ORIGINAL ARTICLE

\title{
Frequency of Hypovitaminosis B12 in Pregnant Women with Gestational Diabetes Mellitus
}

\author{
AESHA SADAF RIZWAN ${ }^{1}$, KOUSAR ROBEEN ${ }^{2}$, AFSHAN AHMAD ${ }^{3}$, IRUM BATOOL HASHMI ${ }^{4}$, MUHAMMAD TAHIR $^{5}$, \\ SABA ABBAS 6 \\ ${ }^{1}$ Assistant Professor Gynaecology and Obstetrics, Shahida Islam Medical and Dental College/ Shahida Islam Teaching hospital, Lodhran \\ Punjab \\ ${ }^{2}$ Senior Registrar Department of Gynaecology and Obstetrics Mohammad College of Medicine/ Muhammad Teaching Hospital, Peshawar \\ ${ }^{3}$ Gynaecologist Cat A Hospital, Batkhela Malakand \\ ${ }^{4}$ Assistant Professor Gynaecology and Obstetrics, Gomal Medical College, D.I.Khan \\ ${ }^{5}$ Assistant Professor of Oncology Allied Hospital/ Faisalabad Medical University, Faisalabad \\ ${ }^{6}$ Senior Registrar, Gynaecology and Obstetrics, Muhammad Teaching hospital, Peshawar \\ Corresponding author: Dr Afshan Ahmad, Email: afshangauhar1@gmail.com, Cell No. +92 3335395341
}

\begin{abstract}
Objective: To determine the frequency of hypovitaminosis B12 in pregnant females presented with gestational diabetes mellitus.

Study Design: Retrospective study

Place and Duration: The department of Gynaecology and Obstetrics, Category A Hospital, Batkhela Malakand and Muhammad Teaching Hospital, Peshawarfor the duration of six months from October 2020 to March 2021.

Methodology: Total 190 pregnant women (95 GDM, 95 non-GDM) were enrolled in this study. Patient's ages were ranging between 18 to 40 years. Detailed demographics including age, gestational age, body mass index (BMI), and gravidty were recorded after taking informed written consent. Blood samples were taken from all the patients for measuring vitamin B12 status. Hypovitaminosis B12 was defined as <300 pg/ml. Data was analyzed by SPSS 24.0.

Results: Mean age of patients was $32.46 \pm 6.82$ years. Mean BMI was $26.24 \pm 2.34 \mathrm{~kg} / \mathrm{m}^{2}$. Mean gestational age was $34.56 \pm 2.18$ weeks. 105 (55.26\%) women were multigravida. 80 (42.11\%) patients had vitamin B12 level $<300 \mathrm{pg} / \mathrm{ml}$. Patients with GDM had high rate of vitaminosis B12 deficiency as compared to non-GDM patients $(65.26 \%$ Vs $18.95 \%)$. A significant association was observed between GDM and hypovitaminosis B12 with pvalue $<0.0001$.

Conclusion: Vitamin B12 deficiency was significantly associated with gestational diabetes mellitus with frequency of $65.26 \%$.

Keywords: Vitaminosis B12, Gestational Diabetes Mellitus, Pregnancy
\end{abstract}

\section{INTRODUCTION}

Pregnancy is often associated with gestational diabetes mellitus. In spite of the fact that GDM affects around 15 percent of pregnant women worldwide [1], its incidence in Asia might approach 17-20 percent [2, 3]. Impaired glucose intolerance and insulin resistance during pregnancy [4] are classified as GDM, which has a number of deleterious effects on both the mother and the child. [5] In the postpartum phase, GDM is related with increased incidence of preeclampsia and cesarean births, shoulder dystocia, and type 2 diabetes mellitus in women. The risk of developing obesity, impaired glucose tolerance (IGT), and type 2 diabetes in childhood or early adulthood is also higher in children born to women with GDM [6].

Biochemical processes such as nuclear maturation and metabolic responses require vitamin B12. Methylcobalamin, S-adenosylmethionine (SAM) and methionine regeneration require vitamin B12 [7]. For DNA methylation, nuclear maturation, and cell division, Methyl Cobalamin is a key methyl donor with one carbon atom. Today, vitamin B12 insufficiency is a growing health concern [8].

There's a connection between hypovitaminosis B12 and future glucose intolerance, insulin resistance (IR), gestational diabetes, and T2DM [9]. GDM-related hypovitaminosis B12 in pregnant women can lead to a variety of congenital abnormalities and birth problems, including intrauterine growth retardation (IVGR) and brain retardation as well as myelination and neural tube defects (NTD) [10]. It also puts women at risk for pre-eclampsia and eclampsia as well as abortion. Low B12 levels in pregnant women are more common due to their low nutritional status, increased vitamin demand and inadequate food supply. As embryonic tissue grows rapidly, it requires more vitamins, leading to a vitamin deficit. Pregnant women with gestational diabetes mellitus (GDM) and the general population suffer from hypovitaminosis B12. The medical literature is full with warnings about vitamin and mineral deficiencies, with hypovitaminosis B12 being a significant hazard to both mother and baby [1112].The present study was conducted with aimed to determine the frequency of vitamin B12 deficiency in pregnant women presented with gestational diabetes mellitus.

\section{MATERIALS AND METHODS}

This retrospective study was conducted at department ofGynaecology and Obstetrics, Category A Hospital BatkhelaMalakand and Muhammad Teaching Hospital, Peshawarfor the duration of six months from October 2020 to March 2021. Total 190 pregnant women were enrolled in this study. Among them 95 women (cases) had gestational diabetes mellitus and 95 non-GDM women taken as control. Patient's ages were ranging between 18 to 40 years. Detailed demographics including age, gestational 
age, body mass index (BMI), and gravidty were recorded after taking informed written consent. Pregnant women with ages $<18$ years, Anemic women, patients with renal failure and those with no written consent were excluded.

$5 \mathrm{ml}$ blood was taken from peripheral vein; $3 \mathrm{ml}$ was taken in EDTA tube and $2 \mathrm{ml}$ in plain tubes. Sera were taken by centrifuging blood sample (x3000 rpm, 15 min.). Analysis of hemoglobin, Hct, RBC counts and Platelet counts were performed by researcher / consultant Pathologist. Sera were used for the estimation of blood glucose (hexokinase) and HbA1c (Colorimetric method) and vitamin B12 by ELISA assay. Hypovitaminosis B12 was defined as $<300 \mathrm{pg} / \mathrm{ml}$. Vitamin B12 levels were categorized as $\geq 300 \mathrm{pg} / \mathrm{mL}$ - as normal, 200 - $299 \mathrm{pg} / \mathrm{mL}$ as marginal deficiency and $<200 \mathrm{pg} / \mathrm{mL}$ - deficiency.

All the data was analyzed by SPSS 24.0. Mean \pm SD was obtained. Frequencies and percentages were recorded in tabulation form. Chi-square test was applied to determine the association between hypovitaminosis B12 and gestational diabetes mellitus. P-value $<0.05$ was taken as statistical significant.

\section{RESULTS}

Mean age of patients was $32.46 \pm 6.82$ years. Mean BMl was $26.24 \pm 2.34 \mathrm{~kg} / \mathrm{m}^{2}$. Mean gestational age was $34.56 \pm 2.18$ weeks. 105 (55.26\%) women had multigravidity while 85 (44.74\%) patients were primigravida. (Table 1)

Table No 1: Baseline details of all the included patients

\begin{tabular}{|l|l|l|}
\hline Variables & Frequency No. & \%age \\
\hline Mean age (yrs) & $32.46 \pm 6.82$ & - \\
\hline Mean BMI (kg/m) & $26.24 \pm 2.34$ & - \\
\hline Gestational age (weeks) & $34.56 \pm 2.18$ & - \\
\hline Gravidity & 105 & 55.26 \\
\hline Multigravida & 85 & 44.74 \\
\hline Primigravida
\end{tabular}

We found that overall $80(42.11 \%)$ patients had vitamin B12 level <300 pg/ml, while 110 (57.89\%) had vitamin B12 level $>300 \mathrm{pg} / \mathrm{ml}$. (Figure 1)

Figure No 1: Overall frequency of vitaminosis B12 deficiency

\section{Vitaminosis B12 Deficiency}

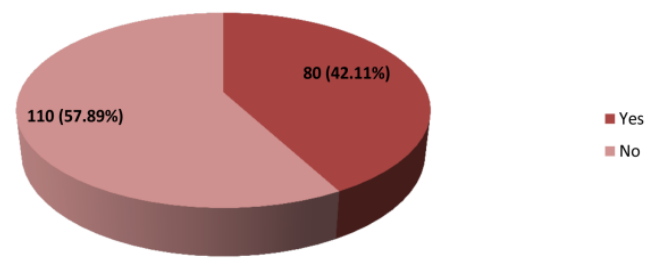

We found that out of 95 patients with GDM $62(65.26 \%)$ patients had vitaminosis B12 deficiency while among 95 non-GDM patients 18 (18.95\%) had vitaminosis B12 deficiency. A significant association was observed between GDM and hypovitaminosis B12 with $p$-value $<0.0001$.

Table No 2: Frequency of Vitaminosis B12 deficiency among cases and control

\begin{tabular}{|c|c|c|c|}
\hline Variable & GDM (n=95) & Non-GDM $(\mathrm{n}=95)$ & P-value \\
\hline \multicolumn{4}{|c|}{ Vitaminosis B12 deficiency } \\
\hline Yes & $62(65.26)$ & $18(18.95)$ & \multirow[t]{2}{*}{$<0.0001$} \\
\hline No & 33 (34.74) & $77(81.05)$ & \\
\hline
\end{tabular}

\section{DISCUSSION}

Gestational diabetes mellitus (GDM) is a condition that affects around $17 \%$ of all pregnancies globally [13]. GDM has been shown to have long-term negative effects on both mothers and their children [14]. Despite the fact that GDM can cause significant problems, the diagnosis is not made until the late second or early third trimester of pregnancy [15]. Vitamin B12 insufficiency has been linked to an increased risk of metabolic abnormalities in pregnant women, including insulin resistance, fatty acid accumulation, and gestational diabetes. [11] Low levels of folate and vitamin B12 during pregnancy increase the chance of having a baby that is too tiny for the mother's gestational age. We conducted present study to determine the frequency of hypovitaminosis B12 in pregnant women presented with gestational diabetes mellitus. In this regard 190 pregnant women were enrolled, out of which 95 with GDM (cases) and 95 were non-GDM taken as control. Mean age of patients in our study was $32.46 \pm 6.82$ years. Mean BMl was $26.24 \pm 2.34 \mathrm{~kg} / \mathrm{m}^{2}$. Mean gestational age was $34.56 \pm 2.18$ weeks. $105(55.26 \%)$ women had multigravidity while 85 (44.74\%) patients were primigravida. These results were comparable to some previous studies in which majority of women were ages between 25 years to 35 years and average BMl was $26.4 \mathrm{~kg} / \mathrm{m}^{2}$ [16-17].

In present study we found that overall 80 (42.11\%) patients had vitamin B12 level $<300 \mathrm{pg} / \mathrm{ml}$, while 110 (57.89\%) had vitamin B12 level $>300 \mathrm{pg} / \mathrm{ml}$. A study conducted by Sukumar $\mathrm{N}$ et al [18] regarding prevalence of vitaminosis B12 deficiency among GDM pregnant women and non-GDM women, in their study overall $26.9 \%$ patients had vitaminosis B12 deficiency.

In our study, we found that out of 95 patients with GDM 62 (65.26\%) patients had vitaminosis B12 deficiency while among 95 non-GDM patients 18 (18.95\%) had vitaminosis B12 deficiency. A significant association was observed between GDM and hypovitaminosis B12 with pvalue $<0.0001$. A meta analysis by Kouroglou $E$ et al [19] regarding association between vitaminosis B12 deficiency and GDM, in which they reported that women with GDM had lower vitamin B12 levels when compared with nonGDM women. Women with vitamin B12 deficiency were at higher risk for developing GDM when compared with those who were vitamin B12 sufficient: OR 1.81 (95\% Cl, 1.252.63, 12: $0 \%$ ). Another study conducted by Xiaotian Chen et al [15] reported that vitamin B12 deficiency was not significantly associated with gestational diabetes mellitus with $p$-value $>0.05$.

A previous study by Ambreenet al 2017 [20] reported hypovitaminosis in $67 \%$ of GDM cases and $39 \%$ in control the findings are in full agreement with the present study. A previous retrospective study from UK reported severe hypovitaminosis B12 in GDM cases compared to nonGDM pregnant women. They further added the risk of GDM is 2.59 times high in hypovitaminosis B12 pregnant women compared to those with normal vitamin B12 [21].

\section{CONCLUSION}

Gestational diabetes mellitus in pregnant women associated with higher rate of maternal and perinatal complications. Vitamin B12 plays an important role in mother and neonatal health. We concluded from this study 
that Vitamin B12 deficiency was significantly associated with gestational diabetes mellitus with frequency of $65.26 \%$.

\section{REFERENCES}

1. Zhu Y, Zhang C. Prevalence of gestational diabetes and risk of progression to type 2 diabetes: a global perspective. Curr Diab Rep. 2016;16(1):7. doi:10.1007/s11892-015-0699-x

2. Zhu WW, Yang HX, Wei YM, et al. Evaluation of the value of fasting plasma glucose in the first prenatal visit to diagnose gestational diabetes mellitus in china. Diabetes Care. 2013;36:586-590. doi:10.2337/dc12-1157

3. Guariguata L, Linnenkamp U, Beagley J, Whiting DR, Cho $\mathrm{NH}$. Global estimates of the prevalence of hyperglycaemia in pregnancy. Diabetes Res Clin Pract. 2014;103(2):176e85. doi:10.1016/j.diabres.2013.11.003.

4. Hod M, Kapur A, Sacks DA, et al. The International Federation of Gynecology and Obstetrics (FIGO) Initiative on gestational diabetes mellitus: a pragmatic guide for diagnosis, management, and care. Int $J$ Gynaecol Obstet. 2015;131(Suppl 3):S173-S211.

5. Farrar D, Simmonds M, Bryant M, et al. Hyperglycaemia and risk of adverse perinatal outcomes: systematic review and meta-analysis. BMJ. 2016;354:i4694. doi:10.1136/bmj.i4694

6. Lowe WL Jr, Scholtens DM, Kuang A, et al. Hyperglycemia and adverse pregnancy outcome follow-up study (HAPO FUS): maternal gestational diabetes mellitus and childhood glucose metabolism. Diabetes Care. 2019;42(3):372-380. doi:10.2337/dc18-1646.

7. Di Cianni G, Lacaria E, Lencioni C, Resi V. Preventing type 2 diabetes and cardiovascular disease in women with gestational diabetes - the evidence and potential strategies. Diabetes Res Clin Pract. 2018;145:184-192.

8. Kalhan SC. One carbon metabolism in pregnancy: Impact on maternal, fetal and neonatal health. Mol Cell Endocrinol (2016) 435:48-60.

9. Lu W-P, Lu M-S, Li Z-H, Zhang C-X. Effects of Multimicronutrient Supplementation during Pregnancy on Postnatal Growth of Children under 5 Years of Age: A MetaAnalysis of Randomized Controlled Trials. PLoS One (2014) 9(2):e88496.

10. Ars CL, Nijs IM, Marroun HE, Muetzel R, Schmidt M, Steenweg-de Graaff J, et al. Prenatal folate, homocysteine and vitamin B12 levels and child brain volumes, cognitive development and psychological functioning: the Generation R Study. Br J Nutr (2016) 122(s1):S1-9.

11. Sukumar N, Rafnsson SB, Kandala N-B, Bhopal R, Yajnik CS, Saravanan P. Prevalence of vitamin B-12 insufficiency during pregnancy and its effect on offspring birth weight: a systematic review and meta-analysis. Am J Clin Nutr (2016) 103(5):1232-51.
12. Lewis, G. The Confidential Enquiry into Maternal and Child Health (CEMACH). In Saving Mothers' Lives: Reviewing Maternal Deaths to Make Motherhood Safer-2003-2005. The Seventh Report on Confidential Enquiries into Maternal Deaths in the United Kingdom; CEMACH: London, UK, 2007.

13. Li Q, Zhang $Y$, Huang $L$, et al. High-dose folic acid supplement use from prepregnancy through midpregnancy is associated with increased risk of gestational diabetes mellitus: a prospective cohort study. Diabetes Care. 2019;42:e113-e115.

14. Rai V. Folate pathway gene MTHFR C677T polymorphism and risk of lung cancer in Asian populations. Asian Pac $J$ Cancer Prev. 2014;15(21):9259-9264.

15. Xiaotian Chen, Yi Zhang, Hongyan Chen, Yuan Jiang, Yin Wang, Dingmei Wang, Mengru Li, Yalan Dou, Xupeng Sun, Guoying Huang, Weili Yan. Association of Maternal Folate and Vitamin $\mathrm{B}_{12}$ in Early Pregnancy With Gestational Diabetes Mellitus: A Prospective Cohort Study. Diabetes Care Jan 2021, 44 (1) 217-223.

16. Yajnik CS, Deshpande SS, Jackson AA, et al. Vitamin B12 and folate concentrations during pregnancy and insulin resistance in the offspring: the Pune Maternal Nutrition Study. Diabetologia. 2008;51:29-38.

17. Liu PJ, Liu Y, Ma L, Yao AM, Chen XY, Hou YX, Wu LP, Xia LY. Associations Between Gestational Diabetes Mellitus Risk and Folate Status in Early Pregnancy and MTHFR C677T Polymorphisms in Chinese Women. Diabetes Metab Syndr Obes. 2020;13:1499-1507.

18. Nithya Sukumar, Sean Wilson, Hema Venkataraman \& Ponnusamy Saravanan. Low vitamin B12 in pregnancy is associated with maternal obesity and gestational diabetes. Endocrine

Abstracts (2015) 38 P206 | DOI: 10.1530/endoabs.38.P206.

19. Kouroglou E, Anagnostis P, Daponte A, Bargiota A. Vitamin B12 insufficiency is associated with increased risk of gestational diabetes mellitus: a systematic review and metaanalysis. Endocrine. 2019 Nov;66(2):149-156.

20. Butt, Ambreen \& Malik, Uzma \& Waheed, Khadija \& Khanum, Amna \& Firdous, Samar \& Ejaz, Sara \& Randhawa, Fawad \& Shakoori, Tania. (2017). Low Serum Cobalamin is a Risk Factor for Gestational Diabetes. Pakistan journal of zoology. 49. 10.17582/journal.pjz/2017.49.6.1963.1968.

21. Saravanan, P., Sukumar, N., Adaikalakoteswari, A. et al. Association of maternal vitamin $B_{12}$ and folate levels in early pregnancy with gestational diabetes: a prospective UK cohort study (PRiDE study). Diabetologia 64, 2170-2182 (2021). 